\title{
ANGIOGENESIS INHIBITION WITH NOVEL COMPOUNDS TARGETING THE KEY GLYCOLYTIC ENZYME PFKFB3
}

\author{
Anahita Abdali ${ }^{1}$, Alberto Corsini ${ }^{1,2}$, Denisa Baci ${ }^{2}$, Carlo De Dominicis ${ }^{3}$, Matteo Zanda ${ }^{3}$, Maria \\ Luisa Gelmi ${ }^{4}$, Stefano Bellosta ${ }^{1,2}$ \\ ${ }^{1}$ Dept. of Pharmacological and Biomolecular Sciences, Università degli Studi di Milano, \\ Milan, Italy \\ ${ }^{2}$ IRCCS MultiMedica, Milan, Italy, \\ ${ }^{3}$ Institute of Medical Sciences, Aberdeen, University of Aberdeen, United Kingdom \\ ${ }^{4}$ Dept. of Pharmaceutical Sciences, Università degli Studi di Milano, Milan, Italy
}

anahita.abdali@unimi.it

Keywords: PFKFB3, glycolysis, MMP, neovessel, endothelial cells

\begin{abstract}
Aim
Intraplaque angiogenesis is associated with progressive and unstable vascular disease. Angiogenesis signals induce endothelial cells (ECs) to switch their metabolism to being highly glycolytic enabling their growth and division. The 6-phosphofructo-2-kinase/fructose-2,6bisphosphatase (PFKFB3) is a key glycolytic activator. PFKFB3 inhibitors have been shown to reduce EC sprouting and seem promising compounds to be used in promoting plaque stability.
\end{abstract}

\section{Methods}

We studied in the human EC line EA.hy926 the effects of 3PO, a commercially available inhibitor of PFKFB3, and of two self-synthesized phenoxindazole analogues (PA-1 and PA-2; based on Boyd et al., 2015) on glycolysis, proliferation, migration, capillary networking, matrix metalloproteinase (MMP) activity and gene expression.

\section{Results}

The three compounds significantly reduced glycolysis levels, while PA-1 and PA-2 affected also capillary-like structure networking. Accordingly, the compounds markedly inhibited EC migration, proliferation and wound closing capacity which are essential for neovessel formation. Moreover, these inhibitors reduced the activity of proMMP-9 and MMP-2 up to 40$50 \%$ and $20-30 \%$ compared to control, respectively, while gelatinase genes expression was downregulated by $80 \%$. Finally, the PA compounds downregulated PFKFB3 expression whilst 3PO did not. Similarly, markers of migration and angiogenesis, such as CCL5, VCAM, VEGFA and VEGFR2, were also markedly reduced (up to 10-fold).

\section{Conclusions}

These findings show that PFKFB3 inhibition with PA compounds interferes with key proangiogenic functions, such as endothelial migration, proliferation and capillary-like structure and molecularly exerts a multitarget anti-angiogenetic activity.

This project has been funded by the European Union's Horizon 2020 Marie Sklodowska Curie grant (\#67552). 